\title{
An analysis of strategic treatment interruptions during imatinib treatment of chronic myelogenous leukemia with imatinib-resistant mutations
}

\author{
Dana Paquin ${ }^{\mathrm{a}, *}$, David Sacco ${ }^{\mathrm{b}}$, John Shamshoian ${ }^{\mathrm{a}}$ \\ a Department of Mathematics California Polytechnic State University, San Luis Obispo, CA 93442, USA \\ ${ }^{\mathrm{b}}$ Department of Mathematics Oklahoma State University, Stillwater, OK 74078, USA
}

\begin{abstract}
A B S T R A C T
Chronic myelogenous leukemia (CML) is a cancer of the white blood cells that results from increased and uncontrolled growth of myeloid cells in the bone marrow and the accumulation of these cells in the blood. The most common form of treatment for CML is imatinib, a tyrosine kinase inhibitor. Although imatinib is an effective treatment for CML and most patients treated with imatinib do attain some form of remission, imatinib does not completely eradicate all leukemia cells, and if treatment is stopped, all patients eventually relapse (Cortes, 2005). In Kim (2008), the authors developed a mathematical model for the dynamics of CML under imatinib treatment that incorporates the anti-leukemia immune response, and in Paquin (2011), the authors used this mathematical model to study strategic treatment interruptions as a potential therapeutic strategy for CML patients. Although the authors presented the results of several numerical simulations in Paquin (2011), the studies in that work did not include the possibility of imatinib-resistant mutations or an initial population of imatinib-resistant leukemia cells. As resistance is a significant consideration in any drug treatment, it is important to study the efficacy of the strategic treatment interruption plan in the presence of imatinib resistance. In this work, we modify the delay differential equations model of Kim (2008), Paquin (2011) to include the possibility of imatinib resistance, and we analyze strategic treatment interruptions as a potential therapeutic tool in the case of patients with imatinib-resistance leukemia cells.
\end{abstract}

\section{Introduction}

Chronic myelogenous leukemia (CML) is a cancer of the blood cells and bone marrow that causes uncontrolled growth of white blood cells. In normal circumstances, the bone marrow makes immature blood stem cells that progress to become mature blood cells. In patients with CML, too many stem cells become white blood cells (called granulocytes, or leukemia cells), due to increased and unregulated growth of myeloid cells in the bone marrow. These cells then accumulate in the blood and bone marrow, and prevent development of healthy white blood cells, red blood cells, and platelets [16].

Drug therapy programs for CML work by targeting the blood cells that contain the abnormal gene responsible for the overabundance of diseased blood cells. In particular, drugs for CML work by targeting the protein produced by this gene, tyrosine kinase. The standard

\footnotetext{
* Corresponding author. Tel.: +18057562679.

E-mail address: dpaquin@calpoly.edu (D. Paquin).
}

treatment for CML is the tyrosine kinase inhibitor imatinib mesylate (marketed as Gleevec or Glivec) [1]. Other targeted drugs for CML treatment include dasatinib and nilotinib. Imatinib does effectively treat CML, and most patients are able to attain some form of remission with continuous, prolonged imatinib treatment. However, imatinib does not completely eradicate all leukemia cells, and if the treatment is stopped, patients eventually relapse $[4,11,15,17]$. Additionally, acquired resistance to imatinib treatment poses a significant problem for CML treatment programs $[13,18]$.

In this work and in other landmark works on imatinib treatment of CML $[11,13-15,17]$, three standard types of remission are considered: hematologic, cytogenetic, and molecular. Each type of remission corresponds to a 2 -log, or 100 -fold, decrease compared to the previous level. According to Lowenberg [9], each patient typically has approximately $10^{12}$ leukemia cells prior to imatinib treatment. Thus, hematologic remission corresponds to $10^{10}$ cells, cytogenetic remission corresponds to $10^{8}$ cells, and molecular remission corresponds to $10^{6}$ cells. Assuming that the average person has approximately $6 \mathrm{~L}$ of blood, these remission levels correspond to the concentration levels given in Table 1. With imatinib treatment, nearly all patients achieve 
Table 1

Leukemia cell concentrations (in $k / \mu \mathrm{L}$ ) corresponding to hematologic, cytogenetic, and molecular remission levels.

\begin{tabular}{llll}
\hline Remission level & Hematologic & Cytogenetic & Molecular \\
\hline Concentration $(k / \mu \mathrm{L})$ & 1.67 & $1.67 \times 10^{-2}$ & $1.67 \times 10^{-4}$ \\
\hline
\end{tabular}

hematologic remission, and approximately $75 \%$ of patients achieve cytogenetic remission $[4,8,13]$.

Several mathematical models have been developed, simulated, and analyzed to study the the pathogenesis, effects, and dynamics of imatinib treatment of CML , including [6,7,11,14,17]. A review of several mathematical models of CML and the clinical insights provided by the models is contained in [13]. In all of the models referenced above, the authors conclude that imatinib does not completely eliminate the leukemia cell population, and propose that imatinib therapy should be combined with an additional form of treatment. In [14], the authors use a stochastic process model to simulate a competition of normal and malignant stem cells for a common resource. In [11], Michor et al. constructed a system of ordinary differential equations to model the dynamics of CML with imatinib treatment. Their model describes the progression of leukemia cells through four different life stages, and stipulates that the progression of leukemia cells from one stage to another is slowed with imatinib treatment. Kim et al. modified this ODE model in [6] to construct a system of delay differential equations that includes not only the progression of leukemia cells through life stages, but also interactions of leukemia cells with antileukemia T-cells. Their work indicates that the strength of a given patient's immune response may be an important factor in determining what type of remission the patient achieves, and how long the patient remains in remission, while undergoing continuous imatinib treatment.

In [12], Paquin et al. used the delay differential equations model presented in [6] to study the effectiveness of strategic treatment interruptions as a potential clinical approach to enhancing the effects of imatinib treatment for CML. The authors demonstrated that treatment programs that involve strategic treatment interruptions in which imatinib treatment is temporarily stopped in order to leverage the antileukemia immune response may prevent leukemia from relapsing and may prevent remission for significantly longer than continuous imatinib treatment, and, in many cases, strategic treatment interruptions may completely eliminate leukemic cells from the body. They also used a series of numerical simulations to determine an optimal time during which imatinib treatment should be temporarily stopped in order to leverage the patient's own anti-leukemia immune response. However, in [12], the authors did not consider the possibility of imatinib resistance. In particular, it was assumed that it is not possible for patients to develop imatinib-resistant leukemia cells, and that patients do not initially have any imatinib-resistant leukemia cells prior to starting treatment. As acquired and innate drug resistance are important considerations in any drug therapy program, the natural next step following the work in [12] is to study the possible efficacy of strategic treatment interruptions when the patient may develop imatinib resistance during treatment.

This paper is organized in the following way. We begin in Section 2 by modifying the delay differential equations model to include the possibility of both pre-treatment imatinib-resistant cancer cells and acquired imatinib resistance. We then derive estimates of the model parameters using experimental data from [3]. We use the universal model parameter values (i.e. the parameter values that are the same for all patients) from [6], and we focus on using the data from [3] to derive new values of patient-dependent parameters in the case of imatinib resistance. In Section 3, we present and analyze the results of several strategic treatment interruption simulations in the presence of imatinib resistance, and we conclude that the success of strategic treatment interruptions in this case is largely dependent on the strength of the patient's immune response. In Section 4, we study the sensitivity of the results to the universal and patient-specific parameters used in the model. Concluding remarks are in Section 5.

\section{A mathematical model for imatinib treatment of CML with imatinib resistance}

In 2005, Michor et al. [11] constructed a differential equations model of CML based on the architecture of the hematopoietic system. In particular, this model describes the development of leukemia cells through four subsequent life stages. The different concentrations of cell populations (in $k / \mu \mathrm{L}$, i.e. thousands of cells per microliter) at time $t$ are denoted as follows: $y_{0}(t)$, leukemia stem cells; $y_{1}(t)$, progenitor cells; $y_{2}(t)$, differentiated cells; and $y_{3}(t)$, terminally differentiated cells. Leukemia cells progress through these different life stages in the following way. Stem cells regenerate themselves at rate $r_{y}$. Stem cells become progenitor cells at rate $a_{y}$ and die at rate $r_{0}$. Progenitor cells become differentiated cells at rate $b_{y}$ and die at rate $d_{1}$. Differentiated cells become terminal cells at rate $c_{y}$ and die at rate $d_{2}$. Terminal cells die at rate $d_{3}$. With imatinib treatment, $a_{y}$ and $b_{y}$ are reduced by a factor of 100 and 750, respectively, thus resulting in a decrease in the overall leukemia concentration.

A second set $z_{0}, z_{1}, z_{2}, z_{3}$ (imatinib-resistant stem, progenitor, differentiated, and terminally differentiated) of leukemia cells was included in this model to account for the possibility of resistance to imatinib. It was assumed that only stem cells can acquire imatinib resistance, and that mutations occur at a rate of $u$ mutations per division.

The system of ODEs that describes the mathematical model of Michor et al. [11] is given below.

$$
\begin{aligned}
\frac{d y_{0}}{d t} & =\left(r_{y}(1-u)-d_{0}\right) y_{0}, & & \frac{d z_{0}}{d t}=\left(r_{z}-d_{0}\right) z_{0}+r_{y} u y_{0}, \\
\frac{d y_{1}}{d t} & =a_{y} y_{0}-d_{1} y_{1}, & \frac{d z_{1}}{d t} & =a_{z} z_{0}-d_{1} z_{1}, \\
\frac{d y_{2}}{d t} & =b_{y} y_{1}-d_{2} y_{2}, & \frac{d z_{2}}{d t} & =b_{z} z_{1}-d_{2} z_{2}, \\
\frac{d y_{3}}{d t} & =c_{y} y_{2}-d_{3} y_{3} . & \frac{d z_{3}}{d t} & =c_{z} z_{2}-d_{3} z_{3} .
\end{aligned}
$$

Kim et al. modified the model of Michor et al. to include the antileukemia immune response. In particular, Kim et al. used experimental T-cell data from [3] to measure the immune response during imatinib treatment of CML. To include the dynamics of the anti-leukemia T-cell response in the mathematical model of CML, Kim et al. added an additional term to each of the equations of the model of Michor et al. to account for the interaction between cancer cells and T-cells that results in death of leukemia cells. In addition, Kim et al. added a delay-differential equation $\frac{d T}{d t}$ to describe the evolution of the T-cell population $T$ during imatinib treatment. The mathematical model of Kim et al. which includes the immune response and imatinib resistance is given by the following:

$$
\begin{aligned}
& \frac{d y_{0}}{d t}=\left(r_{y}(1-u)-d_{0}\right) y_{0}-q_{C} p(C, T) y_{0}, \\
& \frac{d y_{1}}{d t}=a_{y} y_{0}-d_{1} y_{1}-q_{C} p(C, T) y_{1} \\
& \frac{d y_{2}}{d t}=b_{y} y_{1}-d_{2} y_{2}-q_{C} p(C, T) y_{2} \\
& \frac{d y_{3}}{d t}=c_{y} y_{2}-d_{3} y_{3}-q_{C} p(C, T) y_{3} \\
& \frac{d z_{0}}{d t}=\left(r_{z}-d_{0}\right) z_{0}+r_{y} u y_{0}
\end{aligned}
$$


Table 2

Estimates of parameters $[6,11]$.

\begin{tabular}{lll}
\hline Parameter & Description & Estimate \\
\hline$\lambda$ & Fractional adjustment constant & 0.75 \\
$d_{0}$ & Stem cell death rate & $0.003 \lambda /$ day \\
$d_{1}$ & Progenitor cell death rate & $0.008 \lambda$ \\
$d_{2}$ & Differentiated cell death rate & $0.05 \lambda$ \\
$d_{3}$ & Terminal cell death rate & $\lambda$ \\
$r_{y}$ & Stem cell regeneration rate & $0.008 /$ day \\
$a_{y}$ & Stem cell growth rate & 1.6 (without imatinib treatment) \\
& & $1.6 / 100$ (with imatinib treatment) \\
$b_{y}$ & Progenitor cell growth rate & 10 (without imatinib treatment) \\
& & $10 / 750$ (with imatinib treatment) \\
$c_{y}$ & Differentiated cell growth rate & 100 \\
$r_{z}$ & Imatinib resistant mutation stem cell regeneration rate & $0.023 /$ day \\
$a_{z}$ & Imatinib resistant mutation stem cell growth rate & 1.6 \\
$b_{z}$ & Imatinib resistant mutation progenitor cell growth rate & 10 \\
$c_{z}$ & Imatinib resistant mutation differentiated cell growth rate & 100 \\
$k$ & Kinetic (mixing) coefficient & $1(k / \mu \mathrm{L})^{-1}$ per day \\
$p_{0}$ & Probability that T-cell engages cancer cell & 0.8 \\
$q_{C}$ & Probability that cancer cell dies from & 0.75 \\
$q_{T}$ & Probability that T-cell survives encounter with cancer cell & 0.5 \\
$\tau$ & Duration of one T-cell division & 1 day \\
$n$ & Average number of T-cell divisions & Patient-dependent \\
$d_{T}$ & Anti-leukemia T-cell death rate & Patient-dependent \\
$s_{T}$ & Anti-leukemia T-cell supply rate & Patient-dependent \\
$c_{n}$ & Decay rate of immune responsivity & Patient-dependent \\
\hline & &
\end{tabular}

$\frac{d z_{1}}{d t}=a_{z} z_{0}-d_{1} z_{1}$

$\frac{d z_{2}}{d t}=b_{z} z_{1}-d_{2} z_{2}$

$\frac{d z_{3}}{d t}=c_{z} z_{2}-d_{3} z_{3}$

$\frac{d T}{d t}=s_{T}-d_{T} T-p(C, T) C+2^{n} p\left(C_{n \tau}, T_{n \tau}\right) q_{T} C_{n \tau}$,

where

$p(C, T)=p_{0} e^{-c_{n} C} k T, \quad C=\sum_{i=0}^{3} y_{i}$,
$C_{n \tau}=C(t-n \tau), \quad T_{n \tau}=T(t-n \tau)$.

The cancer cell concentrations $y_{k}$ and $z_{k}$ in this model are the same as those in the model of Michor et al. The new variable $C$ represents the total concentration of all leukemia cells, and the new variable $T$ represents the concentration of anti-leukemia $T$ cells. The final terms $q_{c} p_{0} e^{-c_{n} c} k T y_{i}$ in each $\frac{d y_{k}}{d t}$ equation follow the law of mass action, where $k T y_{i}$ describes the rate of interaction between anti-leukemia T-cells and the leukemia cell subpopulation $y_{i}$, and $k$ is the mixing coefficient. The coefficient $p_{0}$ is the probability that a T-cell engages the cancer cell upon interaction, and $q_{C}$ is the probability that the cancer cell dies from the T-cell response. Additionally, although the precise mechanism of down-regulation is unknown, it is known that leukemia cells suppress the anti-leukemia T-cell immune response. This model describes this suppression by modeling the probability that a T-cell engages a cancer cell as exponential decay as a function of the cancer concentration. Thus, the probability of that a T-cell engages with and kills a cancer cell is given by $p_{0} e^{-c_{n} C}$, where $c_{n}$ is the rate of exponential decay due to down-regulation.

In the DDE $\frac{d T}{d t}$, the parameter $s_{T}$ is the constant supply rate of T-cells, the parameter $d_{T}$ is the natural death rate of T-cells, and $p(C, T) C$ is the rate at which T-cells engage leukemia cells and commit to $n$ rounds of division. The final term represents the growth of the Tcell population due to division, where $\tau$ is the average duration of one division, and $q_{T}$ is the probability that a T-cell survives the encounter with an activated leukemia cell. Finally, $C_{n \tau}$ and $T_{n \tau}$ are the total can- cer and T-cell concentrations at time $t-n \tau$, respectively. Once a T-cell begins the division process, it exits the collection of active T-cells and re-enters the system $n \tau$ time units later after $n$ divisions.

The estimated values of the universal parameters for this system are given in Table 2 (and are taken from [11] and [6]). The parameters $n, s_{T}, d_{T}$, and $c_{n}$ are patient-dependent parameters, and in [6] the authors used experimental data from Chen et al. [3] to estimate these values for particular patients in the case without imatinib resistance, i.e. $u=0$ and $z_{0}(0)=0$. We will study three patients, labelled P1, P4, and P12, in detail, as these were the patients in the Chen et al. study with the most available data.

In this work, we use the cancer and T-cell data from Chen et al. [3] to derive new values for these patient-dependent parameters in the presence of imatinib resistance. In particular, we set the initial concentration $z_{0}(0)$ to $10^{-9}(k / \mu \mathrm{L})$, which corresponds to an initial resistant stem cell count of approximately 10 cells, and we set $u$, the rate of imatinib-resistant mutations, equal to $4 \times 10^{-8}$ mutations per division. We also note, however, that mathematical modeling and data analysis in [18] suggests that $u$ may be as high as $10^{-4}$, and in future work, we plan to incorporate the models of that work into our modeling work. Using the T-cell data presented in [3] and known information from the immunological literature [2,5,9] about reasonable ranges for the parameters, we simulated the DDE model to obtain new values for the patient-dependent parameters for P1, P4, and P12 in the presence of imatinib resistance. These new parameter values are the primary difference between this model and the studies presented in [6] and [12], as those studies ignored the possibility of imatinib resistance. Our goal in this work is to evaluate the potential efficacy of strategic treatment interruptions as an alternative therapy program for CML treatment in the presence of imatinib resistance. The values of these newly-derived patient-dependent parameters are presented in Table 4.

Finally, steady-state conditions are used to obtain the initial conditions, as described in Table 3.

In Fig. 1, we illustrate the graphs of the solutions of the delaydifferential equations mathematical model 2 with the estimated values of the universal and patient-dependent parameters for P1, P4, and P12, along with the measured data points from [3], in the case of both initial imatinib resistance and a non-zero resistance mutation rate. These solutions represent continuous imatinib treatment. 
Table 3

Initial leukemia and T-cell concentrations [11].

\begin{tabular}{llll}
\hline Population & Value $\left(\frac{k}{\mu \mathrm{L}}\right)$ & Population & Value $\left(\frac{k}{\mu \mathrm{L}}\right)$ \\
\hline$y_{0}(0)$ & Patient-dependent & $z_{0}(0)$ & $1 \times 10^{-9}$ \\
$y_{1}(0)$ & $\frac{a_{y} y_{0}(0)}{d_{1}}$ & $z_{1}(0)$ & $\frac{a_{z} z_{0}(0)}{d_{1}}$ \\
$y_{2}(0)$ & $\frac{b_{y} y_{1}(0)}{d_{2}}$ & $z_{2}(0)$ & $\frac{b_{z} z_{1}(0)}{d_{2}}$ \\
$y_{3}(0)$ & $\frac{c_{y} y_{2}(0)}{d_{3}}$ & $z_{3}(0)$ & $\frac{c_{z} z_{2}(0)}{d_{3}}$ \\
$T(0)$ & $\frac{s_{T}}{d_{T}}$ & & \\
\hline
\end{tabular}

As illustrated in Fig. 1, and as discussed extensively in [6] and [12] (in the case without imatinib resistance), continuous imatinib treatment does not completely eliminate leukemia, and all patients eventually relapse with continuous imatinib treatment. Additionally, this model with imatinib resistance predicts an earlier relapse with continuous imatinib treatment as compared to the model simulations that do not include resistance in [6] and [12], as expected.

In [6] and [12], the fluctuations that occur in the anti-leukemia T-cell concentrations corresponding to fluctuations that occur in the leukemia cell concentrations are discussed in great detail. The key observations are as follows:

- Initially, the T-cell concentrations are low, as the immune response is nearly fully repressed by leukemia.

- As imatinib treatment begins to take effect, the leukemia cell concentrations decrease, and the patient's immune response is eventually stimulated and the T-cell concentrations increase.

- As the patient's immune response combines with imatinib treatment to combat leukemia, the leukemia cell concentrations decrease, and eventually the T-cells are no longer stimulated and their concentration begins to decrease.

From these observations, we conclude that there is a critical time period during which the patient's immune response is maximally activated, and we seek to devise treatment strategies that optimally leverage the immune response. In [12], the authors studied a wide range of strategic treatment interruptions (STIs) in which imatinib treatment is temporarily stopped in order to stimulate the immune response. The length and starting time of the treatment interruption were varied, and the authors measured the maximum leukemia concentration observed after the STI is administered, the minimum total leukemia concentration observed during treatment, the time required for the patient to achieve cytogenetic remission, and the time required for total cancer elimination (if total elimination occurs). However, the authors did not consider the possibility of imatinib resistance in the initial study.

\section{Results}

In this work, we study the efficacy of numerous strategic treatment interruption simulations in which we incorporate acquired ( $u=4 \times 10^{-8}$ mutations per division) and innate imatinib resistance $\left(z_{0}(0)=1 \times 10^{-9} \mathrm{k} / \mu \mathrm{L}\right)$. In Fig. 2, we plot the leukemia and T-cell concentrations for each patient for $0 \leq t \leq 50$ months, with a 15-day STI starting at time $t=6$ months ( $t=180$ days). We observe that interrupting imatinib treatment results in a 5-fold increase in T-cell concentration as compared to continuous imatinib treatment.

As in [12], we also note that, as a result of the stoppage of imatinib treatment from $t=180$ to $t=195$ days, there is a fast rise in the concentration of leukemia cells while imatinib treatment is stopped. However, this increase does not reach the initial level of leukemia cells present in the patient prior to beginning imatinib treatment. For example, for patient P1, the maximum leukemia concentration
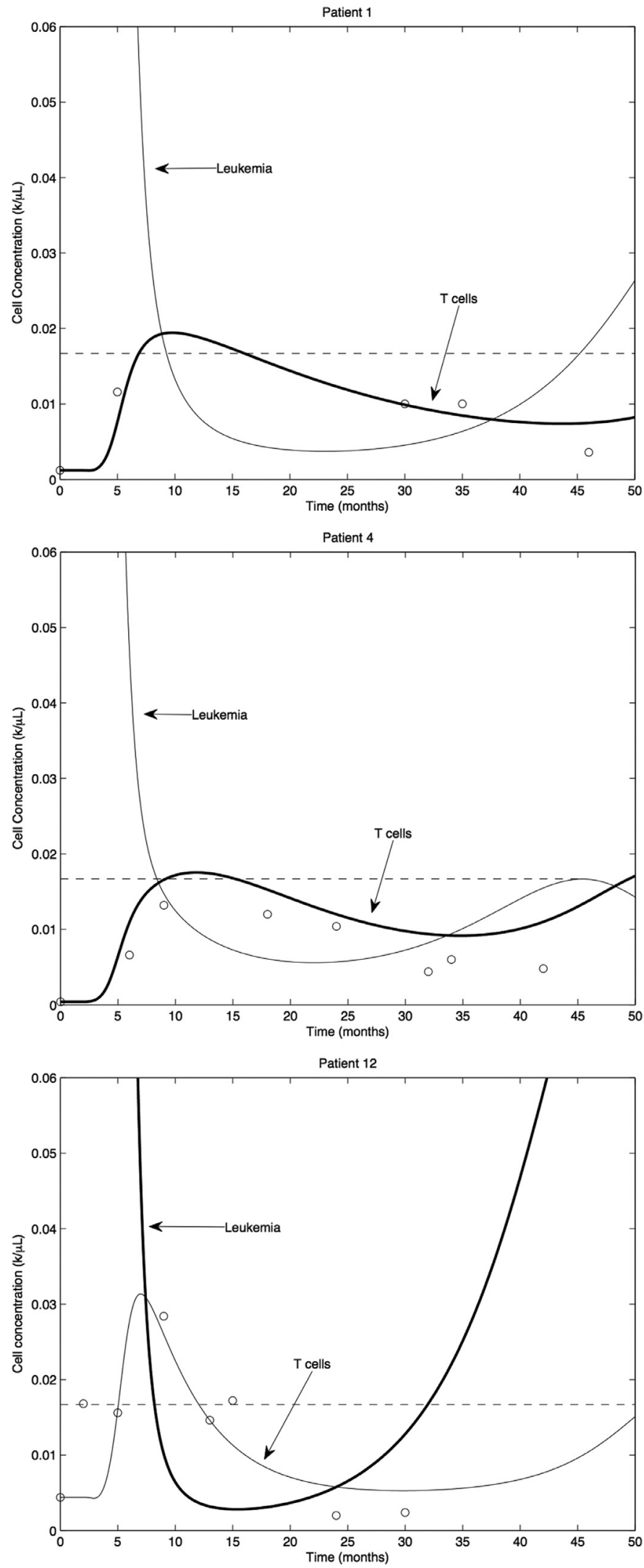

Fig. 1. Model solutions of system (2) fit to T-cell data for patients P1 (top), P4 (middle), and P12 (bottom) in the presence of initial imatinib resistance and non-zero imatinib resistance mutation rate. The numerical values of the parameters are those in Tables 2 and 4. 

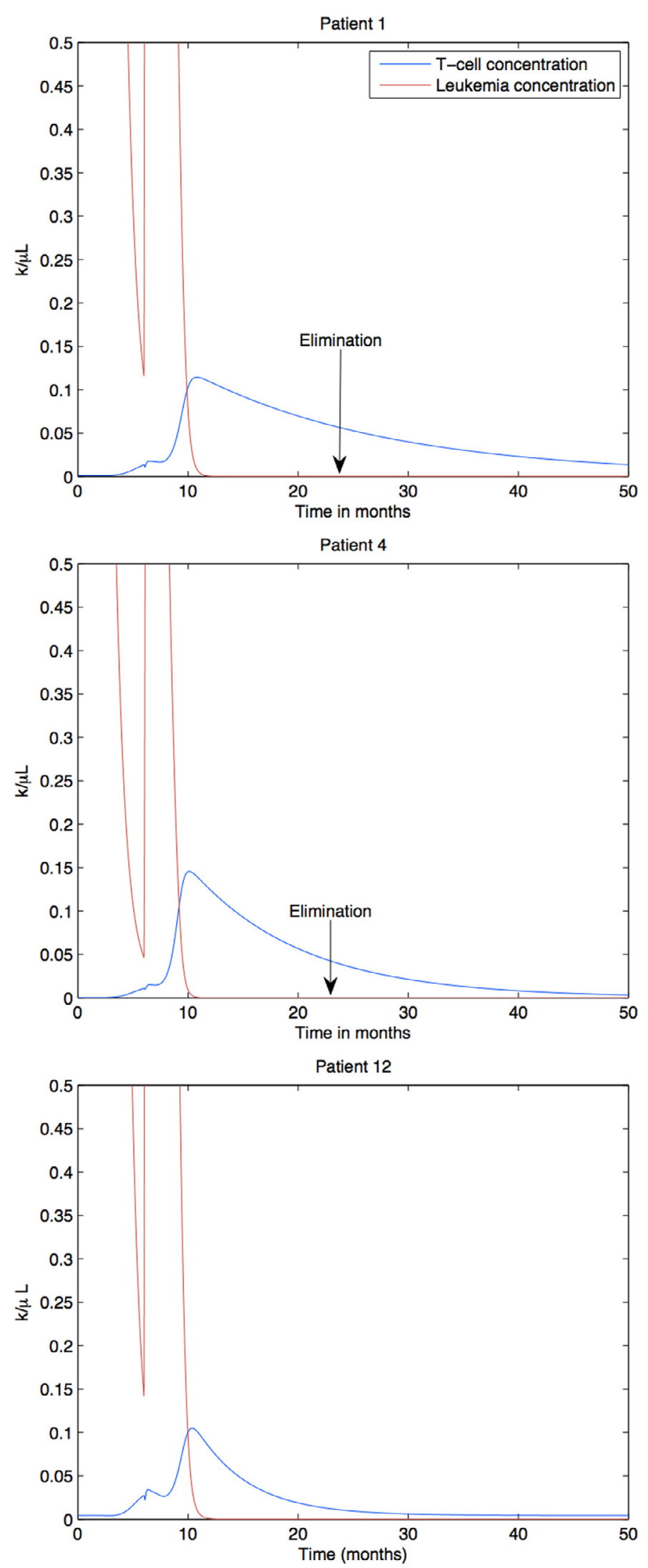

Fig. 2. Leukemia and T-cell concentrations obtained by simulating the model in system (2) for P1, P4, and P12 for $0 \leq t \leq 1500$ days with a 15-day STI from $t=180$ to $t=195$ days with imatinib resistance. attained after imatinib treatment is stopped is $11.00 \mathrm{k} / \mu \mathrm{L}$; the initial pre-treatment leukemia concentration for patient P1 is $73.0 \mathrm{k} / \mu \mathrm{L}$. For all patients, the increase in leukemia concentration that occurs following the stoppage of imatinib treatment does not approach the initial pre-treatment leukemia concentrations. See Table 4 for the pretreatment leukemia concentrations for each patient. Once imatinib treatment is restarted, the leukemia concentration decreases rapidly, and for patients P1 and P4, leukemia is completely eliminated as a result of the STI. We also note that the peaks after treatment is interrupted are higher than the peaks in the non-resistant case discussed in [12], so studying the effects of imatinib resistance is an important consideration when evaluating strategic treatment interruptions as a possible alternative therapy program for CML patients.

In Tables 5-7, we present the results of several 15-day treatment interruptions for patient $\mathrm{P} 1, \mathrm{P} 4$, and $\mathrm{P} 12$ with varying starting times. For each STI, we report the maximum leukemia concentration observed after the STI is administered, the minimum total leukemia concentration observed during treatment, the time required for the patient to achieve cytogenetic remission, and the time required for total cancer elimination (if total elimination occurs), as in [12].

Although patients P1 and P4 achieve complete leukemia elimination with several 15-day strategic treatment interruptions (as was the case in [12] without imatinib resistance), we observe that patient P12 never achieves leukemia elimination with any 15-day STI. The minimum leukemia concentrations presented in Tables 5-7 are much higher than the minimum leukemia concentrations that are achieved in [12] in which imatinib resistance is ignored. Thus, a patient with resistant cells will have a lower chance of complete elimination than a patient with no imatinib-resistant leukemia cells.

The observation that patient P12 does not attain leukemia elimination with any 15-day STI represents a significant difference from the results obtained in [12] without imatinib resistance, as all patients achieved leukemia elimination with a 15-day STI when the possibility of imatinib resistance was ignored. Thus, the case of patient P12 illustrates the importance of considering the possibility of innate and/or acquired imatinib resistance. Patient P12 has an initial leukemia concentration that is an order of magnitude greater than the initial concentrations of the other two patients; we believe that this may be an important factor that affects whether or not leukemia can be eliminated with a single treatment interruption. We also note (but do not include the numerical results here) that patient P12 also does not achieve elimination with any 30-day or 45-day STI.

However, two well-timed strategic treatment interruptions do lead to leukemia elimination for patient P12. As illustrated in Fig. 3, if we interrupt imatinib treatment at $t=150$ days for 30 days and again at $t=390$ days for 60 days, patient P12 will reach a minimum leukemia concentration of $1.40 \times 10^{-6} \mathrm{k} / \mu \mathrm{L}$, which is below the elimination criteria. In Fig. 3, we observe two peaks of leukemia cells and two corresponding T-cell peaks, corresponding to the two separate treatment interruptions.

These results illustrate that strategic treatment interruptions may present a viable alternative therapy program for chronic myelogenous leukemia, even when the patient has an initial count of imatinibresistant leukemia cells and/or a non-zero resistance mutation rate. However, as indicated by the results for P12, the patient-dependent parameters play an important role in whether or not leukemia can be eliminated, and more than one treatment interruption and/or interruptions of various durations may be required to achieve elimination. In the future, we plan to study this optimization problem in more detail as a formal optimal control problem.

\section{Sensitivity analysis}

To study the sensitivity of our results on the numerical values of the parameters and patient-dependent initial conditions in 
Table 4

Values of patient-dependent parameters obtained with imatinib-resistant mutations and initial imatinib-resistant cells: $u=4 \times 10^{-8}$ mutations per division and $z_{0}(0)=1 \times 10^{-9} \mathrm{k} / \mu \mathrm{L}$. These new values represent a major advancement of this work, as earlier work with this model in [6] and [12] did not consider the possibility of imatinib resistance.

\begin{tabular}{lllllll}
\hline Patient & $n$ & $d_{T}$ & $s_{T}$ & $c_{n}$ & $y_{0}(0)$ & $\begin{array}{l}\text { Initial total leukemia } \\
\text { concentration }(k / \mu \mathrm{L})\end{array}$ \\
\hline P1 & 1.195 & 0.0016 & $2.28 \times 10^{-6}$ & 0.85 & $7.6 \times 10^{-6}$ & 73.0 \\
P4 & 1.495 & 0.0033 & $1.34 \times 10^{-6}$ & 2.00 & $2.4 \times 10^{-6}$ & 23.1 \\
P12 & 1.1685 & 0.007 & $3.08 \times 10^{-5}$ & 0.80 & $1.2 \times 10^{-5}$ & 116.8 \\
\hline
\end{tabular}

Table 5

Results of several 15-day STI plans for patient P1 with imatinib resistance.

\begin{tabular}{lllll}
\hline $\begin{array}{l}\text { Treatment } \\
\text { plan: first } \\
\text { day of } \\
\text { 15-day STI }\end{array}$ & $\begin{array}{l}\text { Maximum leukemia } \\
\text { concentration } \\
(k / \mu \mathrm{L}) \text { after STI } \\
\text { is administered }\end{array}$ & $\begin{array}{l}\text { Minimum } \\
\text { leukemia } \\
\text { concentration } \\
(k / \mu \mathrm{L})\end{array}$ & $\begin{array}{l}\text { Time until } \\
\text { cytogenetic } \\
\text { remission } \\
\text { (days) }\end{array}$ & $\begin{array}{l}\text { Time until } \\
\text { total cancer } \\
\text { elimination } \\
\text { (days) }\end{array}$ \\
\hline $\begin{array}{l}\text { No STI } \\
90\end{array}$ & n/a & $3.70 \times 10^{-3}$ & 278 & n/a \\
120 & $\begin{array}{l}20.61 \text { at } t=106 \\
17.22 \text { at } t=136\end{array}$ & $6.67 \times 10^{-3}$ & 339 & n/a \\
150 & 14.29 at $t=166$ & $4.07 \times 10^{-10}$ & 310 & n/a \\
180 & 11.00 at $t=196$ & $1.22 \times 10^{-13}$ & 317 & n/a \\
210 & 7.91 at $t=227$ & $7.06 \times 10^{-16}$ & 331 & 639 \\
240 & 5.55 at $t=257$ & $3.16 \times 10^{-17}$ & 348 & 613 \\
270 & 3.92 at $t=287$ & $2.09 \times 10^{-17}$ & 368 & 624 \\
300 & 2.83 at $t=317$ & $3.24 \times 10^{-16}$ & 278 & 675 \\
330 & 2.11 at $t=347$ & $3.07 \times 10^{-14}$ & 278 & 770 \\
360 & 1.62 at $t=377$ & $3.58 \times 10^{-12}$ & 277 & 933 \\
390 & 1.28 at $t=407$ & $2.30 \times 10^{-10}$ & 277 & n/a \\
\hline
\end{tabular}

Tables 2 and 4 on the effectiveness of the strategic treatment interruption strategies that we considered in Section 3, we apply the Latin Hypercube sampling (LHS) method described in [10]. The LHS technique provides a method for simultaneously sampling a wide range of parameters and statistically determining the correlation between the values of the parameters and various outcomes. We simulate the delay-differential equation model with various strategic treatment interruptions with randomly sampled sets of parameters; using LHS, the values of the parameters are chosen in such a way that each parameter is well-distributed over its range of possible values. In particular, the parameters are sampled uniformly to include the ranges of the parameter values for all patients analyzed in [3], as indicated in Table 9 . We vary every parameter and initial condition used in the model. We perform 500 simulations of strategic treatment interruptions for 15 days for each patient. Times at which the STIs start are $t=90$ to $t=390$ days in 30-day increments.

In Table 8, we report the fraction of LHS samples that result in a successful treatment. A success is defined as a simulation in which the total cancer concentration falls below $10^{-10} \mathrm{k} / \mu \mathrm{L}$ (i.e. a simulation in which the cancer concentration falls below the elimination criterion). We observe a bell-shaped pattern for each patient; peak success rates are for 15-day STIs that start at month 7, 6, and 5 for patients $\mathrm{P} 1, \mathrm{P} 4$, and $\mathrm{P} 12$ respectively. This pattern is due to timing of the peak T-cell expansion rate in conjuction with $\mathrm{T}$-cell stimulation via

Table 6

Results of several 15-day STI plans for patient P4 with imatinib resistance.

\begin{tabular}{llllll}
\hline $\begin{array}{l}\text { Treatment } \\
\text { plan: first } \\
\text { day of } \\
\text { 15-day STI }\end{array}$ & $\begin{array}{l}\text { Maximum leukemia } \\
\text { concentration } \\
(k / \mu \mathrm{L}) \text { after STI } \\
\text { is administered }\end{array}$ & $\begin{array}{l}\text { Maximum } \\
\text { T-cell } \\
\text { concentration } \\
(k / \mu \mathrm{L})\end{array}$ & $\begin{array}{l}\text { Minimum } \\
\text { leukemia } \\
\text { concentration } \\
(k / \mu \mathrm{L})\end{array}$ & $\begin{array}{l}\text { Time until } \\
\text { cytogenetic } \\
\text { remission } \\
\text { (days) }\end{array}$ & $\begin{array}{l}\text { Time until } \\
\text { total cancer } \\
\text { elimination } \\
\text { (days) }\end{array}$ \\
\hline No STI & n/a & $\begin{array}{l}1.75 \times 10^{-2} \text { at } t=354 \\
2.90 \times 10^{-2} \text { at } t=344\end{array}$ & $\begin{array}{l}5.60 \cdot 10^{-3} \\
1.40 \cdot 10^{-3}\end{array}$ & 252 & $\mathrm{n} / \mathrm{a}$ \\
120 & 6.52 at $t=106$ & $\begin{array}{l}6.19 \times 10^{-2} \text { at } t=294 \\
1.11 \cdot 10^{-5}\end{array}$ & 279 & $\mathrm{n} / \mathrm{a} / \mathrm{a}$ \\
150 & 5.45 at $t=136$ & $1.09 \times 10^{-1}$ at $t=292$ & $4.52 \cdot 10^{-9}$ & 282 & $\mathrm{n} / \mathrm{a}$ \\
180 & 4.55 at $t=166$ & $1.46 \times 10^{-1}$ at $t=304$ & $7.14 \cdot 10^{-12}$ & 295 & 688 \\
210 & 3.59 at $t=196$ & $1.71 \times 10^{-1}$ at $t=320$ & $7.94 \cdot 10^{-14}$ & 312 & 581 \\
240 & 2.72 at $t=227$ & $1.86 \times 10^{-1}$ at $t=338$ & $4.55 \cdot 10^{-15}$ & 330 & 557 \\
270 & 2.02 at $t=257$ & $1.89 \times 10^{-1}$ at $t=360$ & $2.47 \cdot 10^{-15}$ & 252 & 573 \\
300 & 1.51 at $t=287$ & $1.78 \times 10^{-1}$ at $t=384$ & $1.53 \cdot 10^{-14}$ & 252 & 617 \\
330 & 1.15 at $t=317$ & $1.59 \times 10^{-1}$ at $t=411$ & $4.39 \cdot 10^{-13}$ & 252 & 702 \\
360 & 0.89 at $t=347$ & $1.37 \times 10^{-1}$ at $t=440$ & $1.92 \cdot 10^{-11}$ & 252 & 870 \\
390 & 0.71 at $t=377$ & $1.17 \times 10^{-1}$ at $t=470$ & $6.38 \cdot 10^{-10}$ & 252 & n/a \\
\hline
\end{tabular}

Table 7

Results of several 15-day STI plans for patient P12 with imatinib resistance.

\begin{tabular}{llllll}
\hline $\begin{array}{l}\text { Treatment } \\
\text { plan: first } \\
\text { day of } \\
\text { 15-day STI }\end{array}$ & $\begin{array}{l}\text { Maximum leukemia } \\
\text { concentration } \\
(k / \mu \mathrm{L}) \text { after STI } \\
\text { is administered }\end{array}$ & $\begin{array}{l}\text { Maximum } \\
\text { T-cell } \\
\text { concentration } \\
(k / \mu \mathrm{L})\end{array}$ & $\begin{array}{l}\text { Minimum } \\
\text { leukemia } \\
\text { concentration } \\
(k / \mu \mathrm{L})\end{array}$ & $\begin{array}{l}\text { Time until } \\
\text { cytogenetic } \\
\text { remission } \\
\text { (days) }\end{array}$ & $\begin{array}{l}\text { Time until } \\
\text { total cancer } \\
\text { elimination } \\
\text { (days) }\end{array}$ \\
\hline $\begin{array}{l}\text { No STI } \\
90\end{array}$ & n/a & $3.13 \times 10^{-2}$ at $t=210$ & $2.80 \times 10^{-3}$ & 246 & $\mathrm{n} / \mathrm{a}$ \\
120 & 27.03 at $t=106$ & $3.29 \times 10^{-2}$ at $t=281$ & $3.80 \times 10^{-3}$ & 320 & n/a \\
150 & 21.35 at $t=166$ & $\begin{array}{l}4.73 \times 10^{-2} \text { at } t=295 \\
1.71 \times 10^{-2} \text { at } t=303\end{array}$ & $7.05 \times 10^{-3}$ & 319 & n/a \\
180 & 13.84 at $t=196$ & $1.05 \times 10^{-1}$ at $t=311$ & $4.27 \times 10^{-6}$ & 320 & n/a \\
210 & 7.66 at $t=227$ & $1.23 \times 10^{-1}$ at $t=320$ & $5.66 \times 10^{-7}$ & 327 & n/a \\
240 & 4.23 at $t=257$ & $1.25 \times 10^{-1}$ at $t=333$ & $2.88 \times 10^{-7}$ & 339 & n/a \\
270 & 2.54 at $t=287$ & $1.04 \times 10^{-1}$ at $t=354$ & $1.53 \times 10^{-6}$ & 246 & n/a \\
300 & 1.69 at $t=317$ & $7.68 \times 10^{-2}$ at $t=381$ & $1.49 \times 10^{-5}$ & 246 & n/a \\
330 & 1.24 at $t=347$ & $5.68 \times 10^{-2}$ at $t=410$ & $8.48 \times 10^{-5}$ & 246 & n/a \\
360 & 0.987 at $t=377$ & $4.35 \times 10^{-2}$ at $t=449$ & $2.70 \times 10^{-4}$ & 246 & n/a \\
390 & 0.838 at $t=407$ & $3.48 \times 10^{-2}$ at $t=469$ & $5.79 \times 10^{-4}$ & 246 & n/a \\
\hline
\end{tabular}




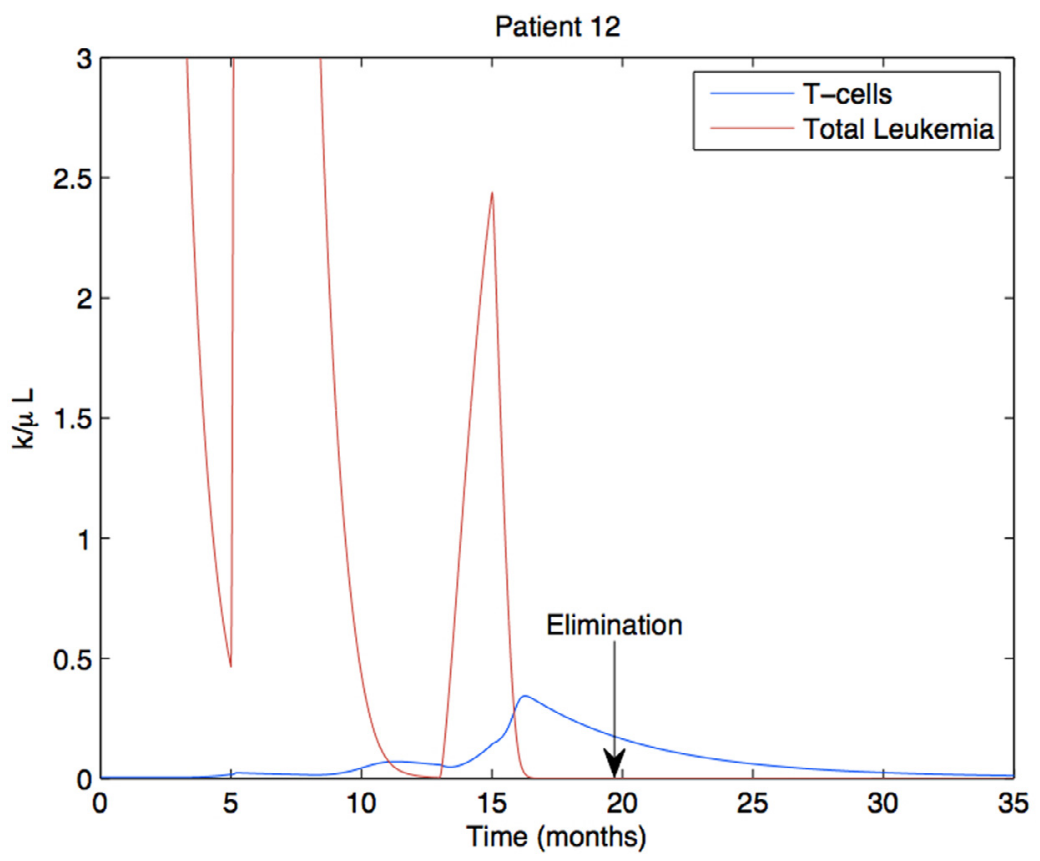

Fig. 3. Leukemia and T-cell concentrations for P12 for $0 \leq t \leq 1050$ days with two STIs, one from $t=150$ to $t=180$ days and a second from $t=390$ to $t=450$ days.

\section{Table 8}

Fraction of LHS samples that result in a successful treatment. A success is defined as a simulation in which the total cancer population falls below $10^{-10} \mathrm{k} / \mu \mathrm{L}$.

\begin{tabular}{llll}
\hline $\begin{array}{l}\text { Treatment } \\
\text { plan: } \\
\text { start of 15-day STI }\end{array}$ & $\begin{array}{l}\text { Fraction of } \\
\text { successful } \\
\text { treatments: P1 }\end{array}$ & $\begin{array}{l}\text { Fraction of } \\
\text { successful } \\
\text { treatments: P4 }\end{array}$ & $\begin{array}{l}\text { Fraction of } \\
\text { successful } \\
\text { treatments: P12 }\end{array}$ \\
\hline 90 & 0.386 & 0.320 & 0.296 \\
120 & 0.440 & 0.396 & 0.346 \\
150 & 0.474 & 0.460 & 0.360 \\
180 & 0.512 & 0.492 & 0.340 \\
210 & 0.520 & 0.474 & 0.308 \\
240 & 0.502 & 0.434 & 0.296 \\
270 & 0.462 & 0.400 & 0.278 \\
300 & 0.446 & 0.368 & 0.274 \\
330 & 0.408 & 0.274 & 0.256 \\
360 & 0.382 & 0.274 & 0.260 \\
390 & 0.368 & 0.264 & 0.256
\end{tabular}

an STI, and in future work we will investigate this relationship more formally.

We also provide in Table 9 the Pearson product-moment correlation (PPMC) coefficient between each parameter and the minimum cancer concentration for patient P1 attained during the course of treatment with a 15-day STI. To obtain the correlation coefficients in Table 9, we performed 1000 LHS simulations with a strategic treatment interruption from $t=210$ to $t=225$ days after the start of treatment, and we note that the correlation coefficients obtained with all other single-interruption STI strategies considered in Section 3 are comparable.

We observe that $n$ and $q_{T}$ are negatively correlated with the minimum leukemia concentration. This negative correlation occurs because the T-cell population expands primarily through interaction with cancer cells, as described by the term $2^{n} p\left(C_{n \tau}, T_{n \tau}\right) q_{T} C_{n \tau}$ in the DDE model. We also observe that the birth rate $r_{z}$ of imatinib-resistant leukemia cells is correlated with the minimum leukemia concentration by an order of magnitude greater than any other birth rate. This suggests that imatinib-resistant cells play a key role in the effectiveness of an STI.
Table 9

Sensitivity analysis of parameters performed for patient P1 for a 15-day strategic treatment interruption from $t=210$ to $t=225$. For each parameter, we report the estimate of the parameter, the range used for Latin Hypercube sampling, and the Pearson product-moment correlation (PPMC) coefficient between the parameter and the minimum cancer concentration. To obtain the correlation coefficients, we performed 1000 simulations in which every parameter was varied over the given range.

\begin{tabular}{|c|c|c|c|}
\hline Parameter & Estimate & Range & PPMC \\
\hline$\lambda$ & 0.75 & 0.5 to 1 & -0.3577 \\
\hline$d_{0}$ & $0.003 \lambda /$ day & $\pm 25 \%$ & -0.2768 \\
\hline$d_{1}$ & $0.008 \lambda$ & $\pm 25 \%$ & -0.3265 \\
\hline$d_{2}$ & $0.05 \lambda$ & $\pm 25 \%$ & -0.3725 \\
\hline$d_{3}$ & $\lambda$ & $\pm 25 \%$ & -0.3190 \\
\hline$r_{y}$ & 0.008 & $\pm 25 \%$ & 0.0486 \\
\hline$a_{y}$ & $\begin{array}{l}1.6 \text { without imatinib } \\
1.6 / 100 \text { with imatinib }\end{array}$ & $\pm 25 \%$ & 0.0385 \\
\hline$b_{y}$ & $\begin{array}{l}10 \text { without imatinib } \\
10 / 750 \text { with imatinib }\end{array}$ & $\pm 25 \%$ & 0.0658 \\
\hline$c_{y}$ & 100 & $\pm 25 \%$ & -0.0062 \\
\hline$r_{z}$ & 0.01 & $\pm 25 \%$ & 0.1233 \\
\hline$a_{z}$ & 1.6 & Same as $a_{y}$ & 0.0385 \\
\hline$b_{z}$ & 10 & Same as $b_{y}$ & 0.0658 \\
\hline$c_{z}$ & 100 & Same as $c_{y}$ & -0.0062 \\
\hline$u$ & $4 \cdot 10^{-8}$ & $4 \cdot 10^{-8}$ to $8 \cdot 10^{-8}$ & -0.0076 \\
\hline$k$ & $1(k / \mu L)^{-1} /$ day & $\pm 25 \%$ & -0.0544 \\
\hline$p_{0}$ & 0.8 & $\pm 25 \%$ & -0.0011 \\
\hline$q_{C}$ & 0.75 & $\pm 25 \%$ & -0.0019 \\
\hline$q_{T}$ & 0.5 & $\pm 25 \%$ & -0.3187 \\
\hline$\tau$ & 1 day & $12-24$ hours & -0.0163 \\
\hline$n$ & 1.195 & $\pm 25 \%$ & -0.2420 \\
\hline$d_{T}$ & 0.0019/day & $\pm 25 \%$ & -0.0087 \\
\hline$s_{T}$ & $2.28 \cdot 10^{-6} k / \mu L /$ day & $\pm 25 \%$ & 0.0379 \\
\hline$c_{n}$ & 0.85 & $\pm 25 \%$ & -0.0050 \\
\hline$y_{0}(0)$ & $7.6 \cdot 10^{-6}$ & $\pm 25 \%$ & 0.0628 \\
\hline$T(0)$ & 0.0012 & $\pm 25 \%$ & -0.0419 \\
\hline$z_{0}(0)$ & $1 \cdot 10^{-9}$ & $\pm 25 \%$ & 0.0225 \\
\hline
\end{tabular}

\section{Conclusions}

In this paper, we have presented the results of numerous strategic treatment interruption simulations for CML patients with acquired or developed imatinib resistance. Our results demonstrate that 
CML can be eradicated even with pre-existing imatinib-resistant cells and a non-zero imatinib-resistance mutation rate. As expected, we achieved lower leukemia elimination success rates as compared to the case without imatinib resistance studied in [12]. In two of the three patients that we studied in this work, one well-timed STI can be enough to completely eliminate leukemia; in one patient, two welltimed STIs were required. This work demonstrates that imatinib resistance is an important consideration when studying STIs as a possible tool for improving treatment of CML, as the results that we obtained here varied significantly from those obtained without resistance in [12]. The important question remains when to start and end the STI, and how many STIs are required to successfully cure CML with imatinib. It is clear from the sensitivity analysis conducted in Section 4 that the effectiveness of any STI treatment strategy relies on the numerical values of the parameters and initial leukemia concentration levels, including resistant cells and resistance mutation rates. Thus, in a given course of treatment, it is important to estimate the values of these parameters as early and as accurately as possible to implement an STI to optimally leverage the patient's anti-immune response. We should also note that although we do conduct a sensitivity analysis on the parameters used in the model (both the patient-dependent and universal parameters), the primary results in this paper are limited in scope in that our results are based on data from only three patients. Still, this work provides a framework for studying strategic treatment interruptions as a possible therapeutic option for individual patients, and this work demonstrates that such treatment programs have the potential to eliminate leukemia, even in the presence of imatinib resistance.

In future work, we plan to study the optimization problems of how many STIs should occur and when they should begin and end in more detail. In particular, we wish to analytically determine the optimal STI strategy for a given patient based on the specific values of the patient's numerical parameters. We also plan to study the stability of this DDE model in the presence of imatinib resistance. Finally, in this work, we studied the dynamics of the immune response when CML is treated with a single drug; in the future, we plan to study the immune response and possible alternative treatment strategies when additional drugs (such as dastainib or nilotinib) are used in conjunction with imatinib.

\section{Acknowledgments}

The authors wish to thank Peter S. Kim (Faculty of Science, University of Sydney, Sydney, Australia), Peter P. Lee (Division of
Hematology, Department of Medicine, Stanford University, Stanford, California), and Doron Levy (Department of Mathematics and Center for Scientific Computation and Mathematical Modeling (CSCAMM), University of Maryland, College Park, Maryland) for helpful conversations regarding this work.

\section{References}

[1] G. Angstreich, B. Smith, R. Jones, Treatment options for chronic myelogenous leukemia: imatinib versus interferon versus allogeneic transplant, Curr. Opin. Oncol. 16 (2004) 95-99.

[2] R. Antia, C.T. Bergstrom, S.S. Pilyugin, S.M. Kaech, R. Ahmed, J. Theor. Biol. 221 (4) (2003) 585-598.

[3] C. Chen, H. Maecker, P. Lee, Development and dynamics of robust T-cell responses to CML, Blood 111 (11) (2008) 5342-5349.

[4] J. Cortes, M. Talpaz, S. O’Brien, D. Jones, R. Luthra, et al., Molecular responses in patients with chronic myelogenous leukemia in chronic phase treated with imatinib mesylate, Clin. Cancer Res. 11 (2005) 3425-3432.

[5] R.J. DeBoer, D. Homann, A.S. Perelson, Different dynamics of CD4+ and CD8+ T cell responses during and after acute lymphocytic choriomeningitis virus infection, J. Immunol. 171 (8) (2003) 3928-3935.

[6] P. Kim, P. Lee, D. Levy, Dynamics and potential impact of the immune response to chronic myelogenous leukemia, PLoS Comput. Biol. 4 (6) (2008).

[7] N. Komarova, D. Wodarz, Drug resistance in cancer: principles of emergence and prevention, Proc. Natl. Acad. Sci. 102 (2005) 9714-9719.

[8] S. Lee, Chronic myelogenous leukemia, Br. J. Hematol. 111 (2000) 993-1009

[9] B. Lowenberg, Minimal residual disease in chronic myeloid leukemia, New England J. Med. 349 (2003) 1399-1401.

[10] M.D. McKay, W.J. Conover, R.J. Beckman, A comparison of three models for selecting values of input variables in the analysis of output from a computer code Technometrics 21 (1979) 239-245.

[11] F. Michor, T. Hughes, Y. Iwasa, S. Branford, N. Shah, C. Sawyers, M. Nowak Dynamics of chronic myeloid leukemia, Nature 435 (2005) 1267-1270.

[12] D. Paquin, P. Kim, P.P. Lee, D. Levy, Strategic treatment interruptions during imatinib treatment of chronic myelogenous leukemia, 73 (5) (2011) 1082-1100.

[13] I. Roeder, I. Glauche, Pathogenesis, treatment effects, and resistance dynamics in chronic myeloid leukemia-insights from mathematical model analyses, J. Mol. Med. 86 (2008) 17-27.

[14] I. Roeder, M. Horn, I. Glauche, A. Hochhaus, M. Mueller, et al., Dynamic modeling of imatinib-treated chronic myeloid leukemia: functional insights and clinical applications, Nat. Med. 12 (2006) 1181-1184.

[15] D.M. Ross, T.P. Hughes, J.V. Melo, Do we have to kill the last CML cell, Leukemia 25 (2) (2011) 193-200.

[16] C. Sawyers, Chronic myeloid leukemia, New England J. Med. 340 (2000) 1330-1340.

[17] M. Tang, M. Gonen, A. Quintas-Cardama, J. Cortes, H. Kantarjian, et al. Dynamics of chronic myeloid leukemia response to long-term targeted therapy reveal treatment effects on leukemic stem cells, Blood 118 (2011) $1622-1631$.

[18] B. Werner, D. Lutz, T.H. Brúmmendorf, A. Traulsen, S. Balabanov, Dynamics of resistance development to imatinib under increasing selection pressure: a combination of mathematical models and in vitro data, PLoS One 6 (12) (2011). 